\title{
The effect of caries increment on oral health-related quality of life among adolescents in Brazil: a 3-year longitudinal study
}

Janice Simpson de PAULA ${ }^{(a)}$ Lívia Guimarães ZINA(a)

Lisa JAMIESON(b)

Fábio Luiz MIALHE(c)

(a) Universidade Federal de Minas Gerais UFMG, School of Dentistry, Department of Community and Preventive Dentistry, Belo Horizonte, MG, Brazil.

(b) The University of Adelaide, Australian Research Centre for Population Oral Health, Adelaide, Australia.

(c) Universidade Estadual de Campinas - Unicamp, Piracicaba Dental School, Department of Community Dentistry, Piracicaba, SP, Brazil.
Declaration of Interests: The authors certify that they have no commercial or associative interest that represents a conflict of interest in connection with the manuscript.

\section{Corresponding Author:}

Janice Simpson de Paula

E-mail: janicesimpson@ufmg.br

https://doi.org/10.1590/1807-3107BOR-2020.vol34.0107

Submitted: April 14, 2020

Accepted for publication: Juy 14, 2020

Last revision: July 22, 2020
Abstract: This study aimed to assess the effect of caries increment on Oral Health-related Quality of Life (OHRQoL) of Brazilian adolescents and to evaluate the responsiveness of the Child Perceptions Questionnaire $\left(\mathrm{CPQ}_{11-14}\right)$ in this group. A population-based sample of 515 Brazilian 12-year-olds from a large city located in the southeast of Brazil was evaluated according to a random multistage sampling design at baseline and 291 at three years follow-up, using the DMFT index and the $\mathrm{CPQ}_{11-14}$ instrument. To evaluate the responsiveness to change, the measures of effect size and longitudinal construct validity were used. It was verified that OHRQoL among adolescents with DMFT increment across the three years worsened significantly $(p<0.05)$ in relation to their counterparts. The effect size varied from small to moderate. The longitudinal construct validity of $\mathrm{CPQ}_{11-14}$ was satisfactory. Caries increment impacted on OHRQoL of adolescents in Brazil. The $\mathrm{CPQ}_{11-14}$ instrument demonstrated acceptable responsiveness properties.

Keywords: Adolescent; Dental Caries; Incidence; Longitudinal Studies; Quality of Life.

\section{Introduction}

During the last few decades, several studies have focused on subjective perceptions of oral health for clinical trials, epidemiologic research and evaluation of health care programs. ${ }^{1}$ This trend has been supported by increasing awareness of the limitations of normative measures to promote patients' wellbeing and full satisfaction with health care. ${ }^{2,3}$ The construct of Oral Health-related Quality of Life (OHRQoL), and instruments created to assess this, has been investigated as an important contributor to the planning and evaluation of public health and health promotion programs. ${ }^{1,4}$

In this context, special attention has been dedicated to the evaluation of the impact of oral disorders on OHRQoL of children and adolescents using specific instruments. ${ }^{5,6}$ Researchers have emphasized the need for studies investigating OHRQoL in longitudinal studies in order to evaluate how it is impacted by different variables, including dental caries., ${ }^{1,2,7}$ Observational studies are an important category of epidemiological study designs to demonstrate the changes in OHRQoL that occur naturally over time in 
populations, as may be found in the studies with elderly people ${ }^{8}$ and children and adolescents ${ }^{2,79,10,11,12}$ using different assessment tools.

However, to investigate how different variables impact on OHRQoL over time, the OHRQoL instrument needs to demonstrate adequate psychometric properties and responsiveness. Responsiveness relates to the ability of a health status measure to detect change in health status over time., ${ }^{2,13}$ Analysis of responsiveness makes it easier for researchers and policy makers to interpret quality of life scores over time, and to thus determine the magnitude of the change measured by a specific question..$^{13,14}$

Although there have been some publications evaluating longitudinally OHRQoL changes in children and adolescents $2,7,14,15,16,17,18$, few have investigated the responsiveness properties of the $\mathrm{CPQ}_{11-14}$ instrument. ${ }^{2,9,19}$ To our knowledge, there are only three published studies that investigated the responsiveness of $\mathrm{CPQ}_{11-14}$, one that investigated Cambodian children taking part in basic dental care programs ${ }^{19}$, and two that evaluated adolescents in New Zealand. ${ }^{9}$ All studies showed that the responsiveness of $\mathrm{CPQ}_{11-14}$ was satisfactory. However because OHRQoL is mediated by diverse personal, social and environmental factors, it is important to consider the complexity of scores mechanism and the conceptual basis underpinning the instrument in different population groups and cultures. ${ }^{14,20}$

The objective of the present study was to assess the effect of caries increment on Oral Health-related Quality of Life (OHRQoL), measured by the CPQ instrument, of Brazilian adolescents over a 3 year period and to evaluate the responsiveness of the Child Perceptions Questionnaire $\left(\mathrm{CPQ}_{11-14}\right)$ in this group.

\section{Methodology}

This longitudinal study was approved by a Research Ethics Committee of a Brazilian Dental School (Protocol No. 147/2012). Written, informed was provided by all parents/guardians.

Sample size was estimated assuming a 95\% confidence level, with an accuracy of $20 \%$ in the DMFT estimate and a minimum test power of 0.80 $(\beta=0.20)$, with a significance level of $5 \%(\alpha=0.05)$ for medium and large effect sizes. Based on a previous conglomerate analysis of a population-based study, an average DMFT of 2.30, a standard deviation of 2.72 and a design effect (deff) of 2 was considered, requiring a minimum sample size of 268. Considering losses to follow-up, a sample of 51512 -year olds was analysed at baseline (2012). After three years, the re-evaluated sample size was 291, providing a minimum power of 0.80 for hypothesis testing. The principal researcher (who conducted the clinical examinations) returned to the schools, with all participants being again invited to take part in the study.

\section{Measures}

The dental clinical examinations was performed according to the World Health Organization (WHO) recommendations. ${ }^{21}$ All adolescents were assessed in the school environment, under natural light, using CPI probes and plane surface mouth mirrors (Golgran, São Paulo, Brazil). Assessments at both time points were performed by a single, calibrated dentist. A Gold Standard examiner conducted the calibration process, with practical and theoretical phases. A kappa value of over 0.91 was obtained for the intra- and inter- examiner calibration.

The DMFT index (decayed, missing and filled teeth in the permanent dentition) was used to assess caries experience, using WHO criteria. ${ }^{20}$ To compare the impact of DMFT increment on OHRQoL of adolescents, two groups were created: G1 - without DMFT increment; and G2 - with DMFT increment. The DMFT increment was calculated by subtracting the DMFT values at baseline from estimates collected at 3-year follow-up.

OHRQoL was measured using the $C P Q_{11-14}$ instrument. ${ }^{22,23} \mathrm{The}^{\mathrm{CPQ}} \mathrm{Q}_{1-14}$ has 35 questions answered on a Likert scale, ranging from 0 (never) to 4 (every day or almost every day). The sum of responses is calculated for all questions (overall $\mathrm{CPQ}_{11-14}$ ) and for each of the 4 domains (Oral Symptoms - OS, Functional Limitations - FL, Emotional Well-being - EWB, Social Well-being - SWB). Lower values represent better OHRQoL. Global questions related to adolescents' perception of their oral health ("Would you say that the health of your teeth, lips, jaws and mouth is..." - answers from 'excellent' to 'poor') and their 
perception of how their oral condition affects their life overall ("How much does the condition of your teeth, lips, jaws or mouth affect your life overall?" - answers from 'not at all' to 'very much') were also included. The questionnaire was self-administered within the school environment with the help of the researcher. ${ }^{24}$

At the 3-year follow-up, a Global Transition Judgment (GTJ) was included, ${ }^{9,14}$. The specific question was: "Since I examined you at age 12, has the health of your teeth, lips, jaws or mouth changed?" with response options "no change", "worsened" or "improvement". ${ }^{2}$ The GTJ is considered a gold standard for assessing changes in subjective perceptions as regards to OHRQoL, since these measurements are less influenced by an individual's mood ${ }^{14}$.

\section{Data analysis}

Statistical analysis was performed using descriptive and inferential methods. Characteristics of adolescents followed-up and not followed-up were compared (sex, school type, two global question and DMFT index), using, Chi-square test for proportions and Student's- $t$ test for means. Descriptive data (mean and standard deviation) from the DMFT index at baseline and follow up were compared using Paired Student's- $t$ Test.

The $C P Q_{11-14}$ scores were initially tested for normality (Kolmogorov-Smirnov test). The null hypothesis was rejected, with a level of significance lower than 0.01 . In this case, the most appropriate statistical tests are non-parametric, used for samples with asymmetrical score distributions. Thus, the $\mathrm{CPQ}_{11-14}$ scores (domains and overall) for all participants were evaluated over time by the nonparametric Wilcoxon test.

After stratifying the sample into the DMFT increment groups (G1 and G2), the nonparametric Wilcoxon test was used to investigate OHRQoL over time. For comparison between independent groups (G1 and G2) at two timepoints, the nonparametric Mann-Whitney test was applied. Supplementary analyses to support data interpretation were used with the same methodology and using the groups: school type (public and private) and sex (male and female).

To evaluate responsiveness to change, measures of effect size and longitudinal construct validity were used. ${ }^{25,26}$ The effect size measure was adopted to establish the magnitude of change observed in $\mathrm{CPQ}_{11-14}$ over time, ${ }^{25}$ for all participants and for two groups analyses. The formula used for Cohen was applied in this study: ${ }^{27}$ "mean baseline score - mean follow-up score/standard deviation of baseline score". Effect size (magnitude of change) of less than 0.2 is considered small, from 0.3 to 0.7 moderate, with 0.8 or above considered large. ${ }^{27}$

The longitudinal construct validity, according GTJ, was evaluated by means of the Kruskal-Wallis one-way analysis of variance, and subsequent use of Dunn's test for to analyze specific sample pairs in post hoc tests. The mean change in $\mathrm{CPQ}_{11-14}$ scores was evaluated against the GTJ. 2,14,16 According to this methodology, participants who reported worsened OHRQoL over time would present a negative value after subtraction; participants who reported no change would present a value close to zero after subtraction and participants who reported improvement in their OHRQoL would present a positive value after subtraction.

Calculations of sample size and test power were performed using R (R Foundation for Statistical Computing, Vienna, Austria) and $\mathrm{G}^{*}$ Power ${ }^{28}$ programs. Data analysis was conducted with the Statistical Package for the Social Sciences (SPSS, version 23.0, SPSS Inc., Chicago, IL, USA), and a level of significance of 0.05 was used.

\section{Results}

At baseline, 515 participants were recruited from 22 schools. At 3-year follow-up, 291 participants were examined (56.5 per cent response rate). The causes of loss of follow-up were mainly due change of school $(25.8 \%)$ or place of residence $(19.1 \%)$, and an option to drop out of the study (54.6\%).

Table 1 presents participant characteristics between those followed-up at 3-years and that loss to follow-up. The only statistically significant difference was school type $(\mathrm{p}<0.05)$.

The DMFT characteristics of the 291 participants followed-up are shown in Table 2. The mean DMFT increment was 0.8 , with statistically significant differences in both overall DMFT and each constituent part (D, M, F) between baseline and follow up. 
The effect of caries increment on oral health-related quality of life among adolescents in Brazil: a 3-year longitudinal study

Table 1. Comparison of the baseline gender, school type, global questions of $C P Q_{11-14}$ and DMFT index according to the DMFT index of those followed- and not followed-up.

\begin{tabular}{|c|c|c|c|c|c|c|c|}
\hline \multirow{3}{*}{ Variable } & \multirow{2}{*}{\multicolumn{2}{|c|}{$\begin{array}{l}\text { Total } \\
515\end{array}$}} & \multirow{2}{*}{\multicolumn{2}{|c|}{$\begin{array}{c}\text { Followed } \\
291\end{array}$}} & \multirow{2}{*}{\multicolumn{2}{|c|}{$\begin{array}{c}\text { Not followed-up } \\
224\end{array}$}} & \multirow{3}{*}{$p$-value } \\
\hline & & & & & & & \\
\hline & $\mathrm{n}$ & $\%$ & $\mathrm{~N}$ & $\%$ & $n$ & $\%$ & \\
\hline \multicolumn{8}{|l|}{ Sex } \\
\hline Female & 290 & 56.3 & 148 & 51.0 & 142 & 49.0 & $0.1588^{* *}$ \\
\hline Male & 225 & 43.7 & 143 & 63.5 & 82 & 46.5 & \\
\hline \multicolumn{8}{|l|}{ School type } \\
\hline Public & 363 & 70.5 & 238 & 65.6 & 125 & 34.4 & $<0.0001^{* *}$ \\
\hline Private & 152 & 29.5 & 53 & 34.9 & 99 & 65.1 & \\
\hline \multicolumn{8}{|c|}{ Self-rated oral health } \\
\hline Excellent & 66 & 12.8 & 31 & $10.7 \%$ & 35 & 15.6 & $0.0738^{* *}$ \\
\hline Very good & 91 & 17.7 & 49 & $16.8 \%$ & 42 & 18.8 & \\
\hline Good & 179 & 34.8 & 97 & $33.3 \%$ & 82 & 36.6 & \\
\hline \multicolumn{8}{|c|}{ Global questions $C P Q_{11-14}$} \\
\hline Fair & 148 & 28.7 & 91 & $31.3 \%$ & 57 & 25.4 & \\
\hline Poor & 31 & 6.0 & 23 & $7.9 \%$ & 8 & 3.6 & \\
\hline \multicolumn{8}{|c|}{ Global impact of oral health on quality of life } \\
\hline Not at all & 198 & 38.4 & 103 & $35.4 \%$ & 95 & 42.4 & $0.1789^{* *}$ \\
\hline Very little & 144 & 28.0 & 91 & $31.3 \%$ & 53 & 23.7 & \\
\hline Some & 129 & 25.0 & 73 & $25.1 \%$ & 56 & 25.0 & \\
\hline A lot & 33 & 6.4 & 16 & $5.5 \%$ & 17 & 7.6 & \\
\hline \multirow[t]{2}{*}{ Very much } & 11 & 2.2 & 8 & $2.7 \%$ & 3 & 1.3 & \\
\hline & mean & SD* & mean & SD* & mean & SD* & \\
\hline \multicolumn{8}{|l|}{ DMFT index } \\
\hline DMFT & 1.1 & 1.7 & 1.0 & 1.7 & 1.2 & 1.7 & $0.1475^{* * *}$ \\
\hline D & 0.3 & 0.8 & 0.3 & 0.8 & 0.3 & 0.9 & $0.2702^{* * *}$ \\
\hline M & 0.0 & 0.2 & 0.0 & 0.2 & 0.0 & 0.2 & $0.4102^{* * *}$ \\
\hline $\mathrm{F}$ & 0.7 & 1.4 & 0.7 & 1.3 & 0.8 & 1.5 & $0.1722^{* * *}$ \\
\hline
\end{tabular}

Table 2. Mean and standard deviation of clinical data according to the DMFT index at Baseline and Follow-up ( $\mathrm{n}=291$ ).

\begin{tabular}{lcccccccc}
\hline \multirow{2}{*}{ Variable } & \multicolumn{2}{c}{ Decayed } & \multicolumn{2}{c}{ Missing } & \multicolumn{2}{c}{ Filled } & \multicolumn{2}{c}{ DMFT } \\
\cline { 2 - 8 } & Baseline & Follow up & Baseline & Follow up & Baseline & Follow up & Baseline & Follow up \\
\hline Mean & 0.3 & 0.5 & 0.0 & 0.1 & 0.7 & 1.3 & 1.0 & 1.8 \\
Standard deviation & 0.8 & 1.1 & 0.2 & 0.3 & 1.3 & 1.7 & 1.7 & 2.3 \\
p-value* & $\mathrm{p}=0.0005$ & $\mathrm{p}=0.0042$ & $\mathrm{p}<0.0001$ & $\mathrm{p}<0.0001$ \\
\hline
\end{tabular}

*Paired Student's-t Test; Power $>0.99$ for a medium effect size $(\mathrm{dz}=0.5)$.

Table 3 shows a reduction in the overall and domain scores of $\mathrm{CPQ}_{11-14}$ across the 3-year time span, showing an improvement in OHRQoL over time. Changes in overall $\mathrm{CPQ}_{11-14}$ and in the EWB and SWB domains were statistically significant. Effect size scores (ES) were considered small for the domains and overall scores of the $\mathrm{CPQ}_{11-14}$ instrument.
The impact of caries incidence on $\mathrm{CPQ}_{11-14}$ scores is reported in Table 4. It shows the results related to changes in OHRQoL among G1 participants (without DMFT increment) and G2 (with DMFT increment) over three years. Of the 291 individuals re-evaluated at follow-up, 190 (65.3\%) showed no DMFT increment and 101 (34.7\%) presented DMFT 
increment. The three years follow-up analyses in each group demonstrated an improvement in OHRQoL for G1 group (without DMFT increment) and in the SWB domain of G2 group (with DMFT increment). This fact is verified by the statistically significant difference for all $\mathrm{CPQ}_{11-14}$ score reductions, indicating improvement in OHRQoL.

Conversely, there was an observed increase in overall $\mathrm{CPQ}_{11-14}$ scores and in the OS and FL domains scores of $\mathrm{CPQ}_{11-14}$ among $\mathrm{G} 2$ participants'.

When G1 and G2 were compared at Baseline for domains and overall $\mathrm{CPQ}_{11-14}$, there were no statistically significant differences $(p>0.05)$. At 3 -year follow-up, a statistically significant difference in all domains and overall $\mathrm{CPQ}_{11-14}$ was observed. These results demonstrated that DMFT increment has a significant impact on the OHRQoL of adolescents compared with those without DMFT increment.

The effect size for each group is presented in Table 4. The values were considered moderate in the G1 group for overall $\mathrm{CPQ}_{11-14}$ scores and $\mathrm{EWB}$ and SWB domains scores; and small for OS and FL domains. In the G2 group, the effect size for overall score and all domain scores were considered small.

Table 5 presents the longitudinal construct validity using the GTJ responsiveness properties of the $\mathrm{CPQ}_{11-14}$. In the results of difference between means, some "worsened" responses to GTJ presented negative

Table 3. Mean, standard deviation, median, overall scores and by domains $C P Q_{11-14}$ instrument in the at baseline and after 3-year followed up in the overall group of Brazilian adolescents $(n=291)$.

\begin{tabular}{|c|c|c|c|c|c|c|c|c|}
\hline \multirow{2}{*}{ Variable } & \multicolumn{3}{|c|}{ Baseline } & \multicolumn{3}{|c|}{ Follow-up } & \multirow{2}{*}{ p-value ${ }^{1}$} & \multirow{2}{*}{$\mathrm{ES}^{2}$} \\
\hline & Mean (SD) & Median & Range & Mean (SD) & Median & Range & & \\
\hline$C P Q_{11-14}$ (overall score) & $25.5(23.4)$ & 18 & $0-106$ & $20.9(19.7)$ & 15 & $0-90$ & $<0.0001$ & 0.20 \\
\hline \multicolumn{9}{|l|}{ Domains } \\
\hline Oral Symptoms & $5.8(3.9)$ & 5 & 0-24 & $5.5(4.2)$ & 5 & $0-18$ & 0.0821 & 0.08 \\
\hline Functional limitation & $5.2(5.6)$ & 3 & 0-28 & $4.8(5.2)$ & 3 & $0-24$ & 0.2334 & 0.07 \\
\hline Emotional well-being & $8.4(8.7)$ & 5 & $0-35$ & $6.5(7.8)$ & 3 & $0-35$ & $<0.0001$ & 0.22 \\
\hline Social well-being & $6.0(8.0)$ & 3 & 0-38 & $4.2(6.1)$ & 1 & $0-26$ & $<0.0001$ & 0.23 \\
\hline
\end{tabular}

'Wilcoxon test: evaluation of significant difference between baseline and follow-up scores; ${ }^{2}$ Effect size; Power $>0.99$ for a medium effect size $(\mathrm{dz}=0.5)$.

Table 4. Mean, standard deviation, median, range of $C P Q_{11-14}$ and domain scores according to groups of adolescents and the effect size values of $C P Q_{11-14}$ instrument.

\begin{tabular}{|c|c|c|c|c|c|c|c|c|}
\hline \multirow{2}{*}{ Groups } & \multicolumn{3}{|c|}{ Baseline } & \multicolumn{3}{|c|}{ Follow-up } & \multirow{2}{*}{$\begin{array}{l}\text { Intra-group } \\
\text { p-value** }\end{array}$} & \multirow{2}{*}{$\mathrm{ES}^{* * *}$} \\
\hline & Mean (SD) & Median & Range* & Mean (SD) & Median & Range* & & \\
\hline \multicolumn{9}{|l|}{ G1 without DMFT increment } \\
\hline$C P Q_{11-14}$ (overall score) & $25.8(24.2)$ & 17 & $0-106$ & $18.4(18.6)^{\ldots \cdots *}$ & 13 & $0-84$ & $<0.0001$ & 0.3 \\
\hline Oral symptoms & $5.7(4.1)$ & 5 & $0-24$ & $4.8(4.2)^{* * * *}$ & 4 & $0-16$ & 0.0013 & 0.2 \\
\hline Functional limitation & $5.4(5.9)$ & 3 & $0-28$ & $4.3(4.9)^{* * * *}$ & 2 & $0-24$ & 0.0031 & 0.2 \\
\hline Emotional well-being & $8.6(8.9)$ & 5 & $0-35$ & $5.6(7.3)^{*+* *}$ & 2 & $0-32$ & $<0.0001$ & 0.3 \\
\hline Social well-being & $6(8.2)$ & 2 & $0-38$ & $3.7(5.7)^{\cdots \cdots *}$ & 1 & $0-26$ & $<0.0001$ & 0.3 \\
\hline \multicolumn{9}{|l|}{ G2 with DMFT increment } \\
\hline$C P Q_{11-14}$ (overall score) & $24.9(21.9)$ & 19 & 0-94 & $25.6(20.9)^{\ldots . \cdots}$ & 20 & 0-90 & 0.1627 & 0.0 \\
\hline Oral symptoms & $6.0(3.6)$ & 5 & $0-15$ & $6.6(3.9)^{* * * *}$ & 6 & $0-18$ & 0.0945 & 0.2 \\
\hline Functional limitation & $4.7(5.0)$ & 3 & $0-20$ & $5.7(5.6)^{* * * *}$ & 5 & $0-24$ & 0.0330 & 0.2 \\
\hline Emotional well-being & $8.2(8.5)$ & 6 & $0-33$ & $8.2(8.5)^{* * * *}$ & 7 & $0-35$ & 0.4737 & 0.0 \\
\hline Social well-being & $6.1(7.6)$ & 3 & $0-36$ & $5.2(6.8)^{* \ldots * *}$ & 2 & $0-26$ & 0.0397 & 0.1 \\
\hline
\end{tabular}

*range: Minimum value- Maximum value, considering the variation of 0-148 in which 0 is considered good OHRQoL and 100 is considered poor OHRQoL; ${ }^{* *}$ longitudinal intra-group differences: p-value of Wilcoxon non-parametric test; ${ }^{* * *}$ effect size; ${ }^{* * *}$ statistically significant differences $(p<0.05)$ in inter-group differences (Mann-Whitney non-parametric test). Wilcoxon: Power $>0.99$ e Mann-Whitney: Power $=0.97$ for a medium effect size $(\mathrm{dz}=0.5)$. 
Table 5. Mean values of domains (Oral Symptoms, Functional Limitation, Emotional Well-Being and Social Well-Being) and overall change in the $C P Q_{11-14}$ scores according to Global Transition Judgment (GTJ) for two groups of Brazilian adolescents.

\begin{tabular}{|c|c|c|c|c|c|c|}
\hline \multirow{2}{*}{ Groups } & \multirow{2}{*}{$\begin{array}{c}\text { Number of } \\
\text { participants (\%) }\end{array}$} & \multicolumn{5}{|c|}{ Mean of difference (= baseline score-follow-up score) } \\
\hline & & OS & $\mathrm{FL}$ & EWB & SWB & $C P Q_{11.14}$ \\
\hline \multicolumn{7}{|c|}{ G1 without DMFT increment } \\
\hline Total & 190 & 0.9 & 1.1 & 3.0 & 2.4 & 7.4 \\
\hline Worsened & $9(4.7 \%)$ & $-3.3^{a}$ & $-4.1^{\mathrm{a}}$ & $1.3^{\mathrm{ns}}$ & $-0.3^{\mathrm{ns}}$ & $-6.4^{a}$ \\
\hline No change & $94(49.5 \%)$ & $1.1^{\mathrm{ab}}$ & $1.7^{\mathrm{b}}$ & $2.6^{\mathrm{ns}}$ & $2.2^{\text {ns }}$ & $7.6^{b}$ \\
\hline \multirow[t]{2}{*}{ Improvement } & $87(45.8 \%)$ & $1.1^{\mathrm{b}}$ & $1.0^{\mathrm{b}}$ & $3.6^{\mathrm{ns}}$ & $2.8^{\text {ns }}$ & $8.6^{b}$ \\
\hline & $p$-value* & 0.04 & 0.00 & 0.79 & 0.06 & 0.03 \\
\hline \multicolumn{7}{|c|}{ G2 with DMFT increment } \\
\hline Total & 101 & -0.6 & -1.0 & 0.0 & 0.9 & -0.7 \\
\hline Worsened & 23 (22.8\%) & $-2.6^{a}$ & $-3.1^{a}$ & $0.7^{\mathrm{ns}}$ & $0.4^{\mathrm{ns}}$ & $-4.7^{\mathrm{ns}}$ \\
\hline No change & 53 (52.5\%) & $0.3^{b}$ & $-0.4^{b}$ & $-0.9^{\text {ns }}$ & $0.9^{\text {ns }}$ & $-0.2^{\mathrm{ns}}$ \\
\hline \multirow[t]{2}{*}{ Improvement } & $25(24.7 \%)$ & $-0.8^{a b}$ & $-0.4^{a b}$ & $1.5^{\mathrm{ns}}$ & $1.5^{\mathrm{ns}}$ & $1.8^{\mathrm{ns}}$ \\
\hline & $p$-value* & 0.00 & 0.02 & 0.18 & 0.75 & 0.07 \\
\hline
\end{tabular}

Dunn's test (after Kruskal-Wallis test*): different superscript letters denote significant differences between mean according GTJ and superscript (ns) denote no significant differences ( $p>0.05)$. Kruskal-Wallis $G 1$ : Power $=0.92$ e G2: Power $=0.82$ for a medium effect size $(f=0.25)$.

$\mathrm{CPQ}_{11-14}$ scores. For "no change" the scores were generally close to zero and for "improvement" most scores were positive. The responsiveness of $\mathrm{CPQ}_{11-14}$ was considered satisfactory, especially in Oral Symptoms and Functional Limitation domains of $C P Q_{11-14}$ for two groups $(\mathrm{p}<0.05)$. In post hoc test for pairs of GTJ, for the Oral Symptoms and Functional Limitation domains of $\mathrm{CPQ}_{11-14}$ the scores of 'worsened" GTJ always presented a statistically significant difference in comparison with the "no change" and/ or "improvement" answers.

\section{Discussion}

This study investigated changes in OHRQoL of Brazilian adolescents over three years. The results demonstrated longitudinal associations between DMFT increment and decreased OHRQoL, measured through the $\mathrm{CPQ}_{11-14}$ instrument. The responsiveness of $\mathrm{CPQ}_{11-14}$ in detecting $\mathrm{OHRQ}$ LL change was considered acceptable according to effect size parameters and longitudinal construct validity values in the scientific literature. ${ }^{14,20}$

The comparison of results of overall and domain scores of the $\mathrm{CPQ}_{11-14}$ instrument at baseline and 3-year follow up demonstrated that study participants had an improvement in their OHRQoL over time. This reduction in the prevalence of impacts across the whole sample over time was also observed in in the few longitudinal studies already published, ${ }^{16,18,19}$ using the same OHRQoL instrument. One hypothesis, based on the theoretical underpinnings of OHRQoL ${ }^{1,14,25,29}$ is that the improvement may have occurred due to changes in the self-perception of body image, as well as changes in psychological, socioeconomic and environmental factors of adolescents at age 12 and 15, which may have directly influenced on their OHRQoL. 2,710,11,12 In addition, a statistically significant improvement occurred in the emotional and social domains of $\mathrm{CPQ}_{11-14}$, reinforcing this hypothesis.

However, when comparing the $\mathrm{CPQ}_{11-14}$ scores of G1 and G2 over time, a different trend was observed. In the without DMFT increment group, improvement over time in the $\mathrm{CPQ}_{11-14}$ scores were verified. This fact could be expected a priori due to several factors, including the absence of clinical changes and no pain and discomfort related to dental caries over time., ${ }^{2,18}$

On the other hand, worsening $\mathrm{CPQ}_{11-14}$ scores over time were observed among the DMFT increment group. In this group, the Functional Limitation (FL) domain of $\mathrm{CPQ}_{11-14}$ presented worsening over time, with a statistically significant difference between 
the scores on baseline and follow-up. These results contrast with the findings of Page and Thomson ${ }^{2}$ who found that caries experience (DMFS) impacted in oral symptoms and emotional well-being domain scores over time. It should also be considered that the DMFS, used by Page and Thomson, ${ }^{2}$ represents a more detailed evaluation of the caries experience which could influenced the assessments of the changes in the $\mathrm{CPQ}_{11-14}$.

When $\mathrm{CPQ}_{11-14}$ scores of groups are compared over time, it is clear that the increase in DMFT had a significant impact on OHRQoL, as there was a statistically significant difference in all comparisons of $\mathrm{CPQ}_{11-14}$ scores. Therefore, even considering the complexity involved in a OHRQoL measure,,$^{1,3,4,14}$ it is possible to consider that caries experience represents an important predictor of changes in OHRQoL over time.

In relation to responsiveness properties, the effect size results for overall $\mathrm{CPQ}_{11-14}$, and EBW and SBW domains demonstrated that the scores were more responsive in the group without DMFT increment. These findings are in line with a study among Thai students aged 10-14 years, indicating that the use of $\mathrm{CPQ}_{11-14}$ may not be responsive to change or sensitive to the impact of low levels of disease..$^{12}$ In addition, researchers demonstrated that $\mathrm{CPQ}_{11-14}$ may not detect changes in OHRQoL when caries levels are low. 2,19

We observed a small effect size of $C P Q_{11-14}$, following the same trend observed in longitudinal studies of OHRQoL using other instruments. ${ }^{9,1011,14}$ In the present study, the small effect size was observed especially in regard to oral symptom and functional limitation domains, which may have occurred due to the low incidence of caries, thereby reducing the accuracy of the instrument in detecting changes in OHRQoL over the three years.

Our study confirms that $C \mathrm{PQ}_{11-14}$ scores were able to measure OHRQoL changes over time, in the oral symptoms and functional limitations domains according to the GTJ. According to a recent review about the quality of the OHRQoL measures for children, ${ }^{30}$ there are still discussions about the ability of OHRQoL questionnaires to assess longitudinal changes in the perception of children and adolescents. This fact brings to light the need for studies in the area of longitudinal evaluations. The assessment of the OHRQoL is a broad construct and, according to the results obtained, properties of EWB and SWB domains for the DMFT increment group require complex interpretation because this age group experiences significant psychosocial interferences]. ${ }^{12}$

Despite interventional studies demonstrating good responsiveness properties of the $\mathrm{CPQ}_{11-14}$ ,2,79,10,11,12,31 they are short-term studies with patients who were actively seeking dental treatment. The self-perception treatment need was likely high, a fact that has a great influence on the instrument's discriminatory capacity. In the present study, we observed that the responsiveness of $\mathrm{CPQ}_{11-14}$ in relation to caries increment over a longer period of time (three years) presented inconsistencies, corroborating the findings of other studies. ${ }^{14,32}$ Rodd et al. ${ }^{10}$ have previously reported that $\mathrm{CPQ}_{11-14}$ presented limitations related to observational evaluation over longer periods in population-based samples. Thus, we consider that the present study brings an important contribution to this field of knowledge, demonstrating that the instrument may not be the best option for those who wish to assess longitudinal changes in OHRQoL in children and adolescents with low caries incidence. According to a recent systematic review, the incidence of caries is low in children and adolescents, a fact that should guide researchers in future studies with those populations. ${ }^{33}$

This study has some limitations. OHRQoL is a complex construct and influenced by social and environmental factors, variables not measured in our analysis. Moreover, the low prevalence and incidence of dental caries, and the largest number of filled teeth in the studied population may have influenced the psychometric capacity of $\mathrm{CPQ}_{11-14}$ on detecting longitudinal changes and in comparison between groups. This needs to be taken into consideration in interpretation of results and future studies. In the same way, there are other clinical features that might have influenced $\mathrm{CPQ}_{11-14}$ scores, such as fluorosis and malocclusion, and deserve further investigation. Finally, the imbalance of the sample size can be considered as a limitation in the longitudinal construct validity evaluation. After three years, only 
The effect of caries increment on oral health-related quality of life among adolescents in Brazil: a 3-year longitudinal study

9 participants in G1 (without DMFT increment) worsened according to the Global Transition Judgment (GTJ). This promoted an imbalance in the sample size of the groups analyzed.

\section{Conclusion}

It was observed that increasing caries experience in adolescent over time worsened their OHRQoL in comparison to their counterpart. The longitudinal psychometric properties of $\mathrm{CPQ}_{11-14}$ demonstrated a variation between small to moderate effect size and the instrument was considerable acceptable responsive to change.

\section{Acknowledgment}

This study was supported by São Paulo Research Foundation - FAPESP (2011/17669-5 and 2011/01281-8), São Paulo, Brazil.

\section{References}

1. Inglehart MR, Bagramian RA. Oral health-related quality of life. Chicago: Quintessence; 2002.

2. Foster Page LA, Thomson WM. Caries prevalence, severity, and 3 -year increment, and their impact upon New Zealand adolescents' oral-health-related quality of life. J Public Health Dent. 2012;72(4):287-94. https://doi.org/10.1111/j.1752-7325.2012.00336.x

3. Leão A, Sheiham A. Relation between clinical dental status and subjective impacts on daily living. J Dent Res. 1995 Jul;74(7):1408-13. https://doi.org/10.1177/00220345950740071301

4. Gherunpong S, Sheiham A, Tsakos G. A sociodental approach to assessing children's oral health needs: integrating an oral health-related quality of life (OHRQoL) measure into oral health service planning. Bull World Health Organ. 2006 Jan;84(1):36-42. https://doi.org/10.2471/BLT.05.022517

5. Barbosa TS, Gavião MB. Oral health-related quality of life in children: part II. Effects of clinical oral health status. A systematic review. Int J Dent Hyg. 2008 May;6(2):100-7. https://doi.org/10.1111/j.1601-5037.2008.00293.x

6. Paula JS, Leite IC, Almeida AB, Ambrosano GM, Pereira AC, Mialhe FL. The influence of oral health conditions, socioeconomic status and home environment factors on schoolchildren's self-perception of quality of life. Health Qual Life Outcomes. 2012 Jan;10(1):6. https://doi.org/10.1186/1477-7525-10-6

7. Benson PE, Da'as T, Johal A, Mandall NA, Williams AC, Baker SR, et al. Relationships between dental appearance, self-esteem, socio-economic status, and oral health-related quality of life in UK schoolchildren: A 3-year cohort study. Eur J Orthod. 2015 Oct;37(5):481-90. https://doi.org/10.1093/ejo/cju076

8. Gülcan F, Nasir E, Ekbäck G, Ordell S, Åstrøm AN. Change in Oral Impacts on Daily Performances (OIDP) with increasing age: testing the evaluative properties of the OIDP frequency inventory using prospective data from Norway and Sweden. BMC Oral Health. 2014 May;14(1):59. https://doi.org/10.1186/1472-6831-14-59

9. Foster Page LA, Thomson WM, Locker D. Assessing the responsiveness of the CPQ11-14 in NZ adolescents. Soc Sci Dent. 2010;1:48-53.

10. Rodd HD, Marshman Z, Porritt J, Bradbury J, Baker SR. Oral health-related quality of life of children in relation to dental appearance and educational transition. Br Dent J. 2011 Jul;211(2):E4. https://doi.org/10.1038/sj.bdj.2011.574

11. Rodd HD, Marshman Z, Porritt J, Bradbury J, Baker SR. Psychosocial predictors of children's oral health-related quality of life during transition to secondary school. Qual Life Res. 2012 May;21(4):707-16. https://doi.org/10.1007/s11136-011-9967-7

12. Gururatana O, Baker SR, Robinson PG. Determinants of children's oral-health-related quality of life over time. Community Dent Oral Epidemiol. 2014 Jun;42(3):206-15. https://doi.org/10.1111/cdoe.12080

13. Guyatt G, Walter S, Norman G. Measuring change over time: assessing the usefulness of evaluative instruments. J Chronic Dis. 1987;40(2):171-8. https://doi.org/10.1016/0021-9681(87)90069-5

14. Locker D, Jokovic A, Clarke M. Assessing the responsiveness of measures of oral health-related quality of life. Community Dent Oral Epidemiol. 2004 Feb;32(1):10-8. https://doi.org/10.1111/j.1600-0528.2004.00114.x

15. Antunes LA, Andrade MR, Leão AT, Maia LC, Luiz RR. Systematic review: change in the quality of life of children and adolescents younger than 14 years old after oral health interventions: a systematic review. Pediatr Dent. 2013 Jan-Feb;35(1):37-42.

16. Paula JS, Sarracini KL, Meneghim MC, Pereira AC, Ortega EM, Martins NS, et al. Longitudinal evaluation of the impact of dental caries treatment on oral health-related quality of life among schoolchildren. Eur J Oral Sci. 2015 Jun;123(3):173-8. https://doi.org/10.1111/eos.12188

17. Mashoto KO, Astrøm AN, Skeie MS, Masalu JR. Changes in the quality of life of Tanzanian school children after treatment interventions using the Child-OIDP. Eur J Oral Sci. 2010 Dec;118(6):626-34. https://doi.org/10.1111/i.1600-0722.2010.00776.x 
18. Brondani B, Emmanuelli B, Alves LS, Soares CJ, Ardenghi TM. The effect of dental treatment on oral health-related quality of life in adolescents. Clin Oral Investig. 2018 Jul;22(6):2291-7. https://doi.org/10.1007/s00784-017-2328-3

19. Turton BJ, Thomson WM, Foster Page LA, Saub R, Ishak AR. Responsiveness of the Child Perceptions Questionnaire11-14 for Cambodian children undergoing basic dental care. Int J Paediatr Dent. 2015 Jan;25(1):2-8. https://doi.org/10.1111/ipd.12091

20. Sischo L, Broder HL. Oral health-related quality of life: what, why, how, and future implications. J Dent Res. 2011 Nov;90(11):1264-70. https://doi.org/10.1177/0022034511399918

21. World Health Organization - WHO. Oral health surveys: basic methods. 5th ed. Geneva: World Health Organization; 2013.

22. Jokovic A, Locker D, Stephens M, Kenny D, Tompson B, Guyatt G. Validity and reliability of a questionnaire for measuring child oral-health-related quality of life. J Dent Res. 2002 Jul;81 (7):459-63. https://doi.org/10.1177/154405910208100705

23. Barbosa TS, Tureli MC, Gavião MB. Validity and reliability of the Child Perceptions Questionnaires applied in Brazilian children. BMC Oral Health. 2009 May;9(1):13. https://doi.org/10.1186/1472-6831-9-13

24. Ramos-Jorge ML, Vieira-Andrade RG, Martins-Júnior PA, Cordeiro MM, Ramos-Jorge J, Paiva SM, et al. Level of agreement between self-administered and interviewer-administered $C P Q_{8-10}$ and $C P Q_{8-14}$. Community Dent Oral Epidemiol. 2012 Jun;40(3):201-9. https://doi.org/10.1111/j.1600-0528.2011.00652.x

25. Masood M, Masood Y, Saub R, Newton JT. Need of minimal important difference for oral health-related quality of life measures. J Public Health Dent. 2014;74(1):13-20. https://doi.org/10.1111/j.1752-7325.2012.00374.x

26. Revicki D, Hays RD, Cella D, Sloan J. Recommended methods for determining responsiveness and minimally important differences for patient-reported outcomes. J Clin Epidemiol. 2008 Feb;61(2):102-9. https://doi.org/10.1016/i.jclinepi.2007.03.012

27. Cohen J. Statistical power analysis for the behavioural sciences. 2nd ed. Hillsdale (NJ): Lawrence Erlbaum and Associates; 1988.

28. Faul F, Erdfelder E, Lang AG, Buchner A. G*Power 3: a flexible statistical power analysis program for the social, behavioral, and biomedical sciences. Behav Res Methods. 2007 May;39(2):175-91. https://doi.org/10.3758/BF03193146

29. Gilchrist F, Rodd H, Deery C, Marshman Z. Assessment of the quality of measures of child oral health-related quality of life. BMC Oral Health. 2014 Apr;14(1):40. https://doi.org/10.1186/1472-6831-14-40

30. Kumar S, Kroon J, Lalloo R. A systematic review of the impact of parental socio-economic status and home environment characteristics on children's oral health related quality of life. Health Qual Life Outcomes. 2014 Mar;12(1):41. https://doi.org/10.1186/1477-7525-12-41

31. Buzatti BC, Maroneze MC, Ardenghi TM. Responsiveness of the Brazilian versions of CPQ11-14 and Child-OIDP. Int J Paediatr Dent. 2018 Nov;28(6):641-7. https://doi.org/10.1111/ipd.12423

32. Agou S, Malhotra M, Tompson B, Prakash P, Locker D. Is the child oral health quality of life questionnaire sensitive to change in the context of orthodontic treatment? A brief communication. J Public Health Dent. 2008;68(4):246-8. https://doi.org/10.1111/j.1752-7325.2008.00093.x

33. Hummel R, Akveld NA, Bruers JJ, Sanden WJ, Su N, Heijden GJ. Caries progression rates revisited: a systematic review. J Dent Res. 2019 Jul;98(7):746-54. https://doi.org/10.1177/0022034519847953 